\title{
Ehrenamtliche in der Flüchtlingshilfe - sind gesundheitsfördernde Maßnahmen (durch den ÖGD) notwendig?
}

\section{Volunteers in Refugee Help: is Health Promotion (by Public Health Services) Necessary?}

\section{(1) (1) $\circledast$}

\author{
Autoren \\ Valerie Andrees ${ }^{1}$, Beke Freyer ${ }^{1}$, Ingrid Borgmeier ${ }^{2}$, Hans-Jürgen Galle², Joachim Westenhöfer ${ }^{1}$
}

Institute

1 Hochschule fur Angewandte Wissenschaften Hamburg, Competence Center Gesundheit, Department Gesundheitswissenschaften, Hamburg

2 Bezirksamt Hamburg-Bergedorf, Fachamt Gesundheit, Hamburg

\section{Schlüsselwörter}

Ehrenamt, Flüchtlingshilfe, gesundheitliche Belastungen, gesundheitliche Ressourcen

\section{Key words}

Volunteers, health-related strains, health-related resources, refugee help

\section{Bibliografie}

DOI http://dx.doi.org/10.1055/s-0043-121694

Online-Publikation: 13.12.2017

Gesundheitswesen 2018; 80: 286-292

(c) Georg Thieme Verlag KG Stuttgart · New York, ISSN 0941-3790

\section{Korrespondenzadresse}

Prof. Joachim Westenhöfer

Hochschule fur Angewandte Wissenschaften Hamburg

Fakultat Life Sciences

Health Sciences

Ulmenliet 20

21033, Hamburg

joachim@westenhoefer.de

$\oplus$ Den Anhang des Beitrags finden Sie online unter http://dx.doi.org/10.1055/s-0043-121694.

\section{ZUSAMMENFASSUNG}

Zielsetzung Es sollen gesundheitliche Ressourcen und Belastungen von ehrenamtlich in der Flüchtlingshilfe tätigen Helfern untersucht werden.
Methodik Qualitative Interviews mit 10 ehrenamtlich in der Flüchtlingshilfe tätigen Helferinnen und Helfern sowie mit 11 koordinativ tätigen Fachleuten wurden durchgeführt, transkribiert und mittels qualitativer Inhaltsanalyse ausgewertet.

Ergebnisse Die ehrenamtliche Tätigkeit in der Flüchtlingshilfe wird als starke Ressource erlebt. Die Ehrenamtlichen erfahren viel Dankbarkeit und Bestätigung und erleben das Engagement als bereichernd und selbstwert-stärkend. Andererseits werden aber auch viele Belastungen und Probleme erlebt. Schwierigkeiten in der zeitlichen und emotionalen Abgrenzung werden als wichtige Ursache für die erlebten Belastungen benannt.

Schlussfolgerungen Angebote zur Unterstützung der ehrenamtlich in der Flüchtlingshilfe tätigen Personen sollten optimiert und ausgebaut werden, um die Helfer vor gesundheitlichen Belastungen besser zu schützen. Hierbei könnte der öffentliche Gesundheitsdienst wichtige Aufgaben übernehmen.

\section{ABSTRACT}

Objectives To investigate health-related strains and resources of volunteers in refugee help.

Methods Qualitative interviews with 10 volunteers in refugee help as well as 11 interviews with experts involved in coordinative tasks were conducted, transcribed and analysed using qualitative content analysis.

Results Volunteers' work in refugee help is experienced as a strong resource. Volunteers receive a lot of thankfulness and appreciation and experience their work as enriching and enhancing self-worth. On the other hand, they also experience several stresses and problems. Difficulties in setting up appropriate boundaries regarding time and emotional involvement are mentioned as important reasons for the experienced stresses.

Conclusions Support for volunteers in refugee help should be optimised and expanded in order to protect the volunteers from health-related strains. Public Health Services could play an important role in such improvements. 


\section{Einleitung}

In der zweiten Jahreshälfte 2016 waren weltweit rund 65,3 Millionen Menschen auf der Flucht [1]. Auch die Zahl der Flüchtlinge, die nach Deutschland kommen, hat in den vergangenen Jahren stark zugenommen [2-4]. Insgesamt kamen im Jahr 2015 rund 890.000 Flüchtlinge nach Deutschland [3].

Die Zahl der Flüchtlinge stellt die Kommunen und Länder, insbesondere das Sozial-, Bildungs-, und Gesundheitssystem in Deutschland vor große Herausforderung, bietet aber auch große Chancen [5]. Für die humanitäre Hilfe einschließlich der Gesundheitsversorgung existieren internationale Mindeststandards [6], die auch in der gegenwärtigen Flüchtlingssituation in Deutschland anzuwenden sind, die aber offenbar nicht immer allen Akteuren bekannt sind [7]. Zu den Herausforderungen und Möglichkeiten der gesundheitlichen Versorgung ist im letzten Jahre eine breite wissenschaftliche Diskussion entstanden [8-10].

Im Zusammenhang mit dem enormen Zustrom von Flüchtlingen engagierten sich viele Menschen ehrenamtlich in verschiedenen Bereichen der Flüchtlingshilfe [11]. Eine Analyse von Netzwerkstrukturen in der Flüchtlingsarbeit in der Stadt Erlangen kam zu dem Ergebnis, „dass das Engagement ehrenamtlicher Akteure das Rückgrat der Flüchtlingsarbeit ... darstellt“ [12]. Ehrenamtliche wirken als Bindeglied zwischen Flüchtlingen und Aufnahmegesellschaft und tragen dazu bei, dass Flüchtlinge sowohl kurzfristig als auch auf lange Sicht gesehen erfolgreich integriert werden können $[5,8,13]$.

Die Begriffe Ehrenamt, Freiwilligenarbeit, bürgerschaftliches Engagement und freiwilliges Engagement stehen in einem engen Zusammenhang und werden weitgehend synonym verwendet [14]. Umschrieben wird damit das (zumeist) freiwillige Engagement der Bürger in Vereinen, Initiativen, Gruppen, Organisationen sowie öffentlichen Institutionen und Einrichtungen [15], also in einem festen organisatorischen Rahmen. Die freiwillige bzw. ehrenamtliche Tätigkeit wird in der Regel nebenberuflich durchgeführt und dient nicht zur Bestreitung des Lebensunterhalts, auch wenn eine Aufwandsentschädigung gezahlt wird [16]. In der vorliegenden Arbeit werden die Begriffe Ehrenamt und Freiwillige im Folgenden synonym gebraucht.

Die Aufgaben, die von ehrenamtlichen Helfern in der Flüchtlingshilfe übernommen werden, sind sehr vielseitig und hängen individuell stark von den Ressourcen der Engagierten und dem Bedarf an Unterstützung ab. Ehrenamtliche Helfer können diese wichtigen Funktionen allerdings nur dann ausfüllen, wenn ihre Gesundheit erhalten bleibt $[17,18]$.

\section{Ehrenamt und Gesundheit}

Der Zusammenhang von Ehrenamt und Gesundheit wurde in verschiedenen Studien untersucht. Diese zeigten übereinstimmend, dass ein Ehrenamt einen positiven Effekt auf die Gesundheit von Ehrenamtlichen ausübt [19-22].

Die Ausführung eines Ehrenamtes kann jedoch auch negative Effekte haben. Die Wahrscheinlichkeit, ein Burnout-Syndrom zu erleiden, ist bei Ehrenamtlichen höher als bei professionell Tätigen; dies gilt insbesondere für Menschen, die eine hohe emotionale Last tragen, deren Erwartungen nicht erfüllt werden oder die sich nicht gut auf ihre Tätigkeit vorbereitet fühlen [19].

\section{Fragestellung und Zielsetzung}

Die ehrenamtliche Arbeit in der Flüchtlingshilfe ist nur eingeschränkt vergleichbar mit ehrenamtlichen Tätigkeiten in anderen Bereichen (z. B. in einem Sportverein). Der Großteil der Tätigkeiten in der ehrenamtlichen Flüchtlingshilfe findet in engem Kontakt mit Flüchtlingen statt, welche teilweise traumatisierende Erlebnisse durchlebt haben. Die Konfrontation und Auseinandersetzung mit solchen Erlebnissen stellt für die Helfer eine zusätzliche Belastung dar. Allerdings gibt es bislang keine Studien, die die gesundheitlichen Belastungen, aber auch nutzbare Ressourcen von Ehrenamtlichen in der Flüchtlingshilfe erfassen. Vor diesem Hintergrund untersucht die vorliegende Arbeit die Frage, welche gesundheitlichen Auswirkungen die ehrenamtliche Tätigkeit in der Flüchtlingshilfe hat und wie die Ehrenamtlichen gegebenenfalls besser unterstützt werden können. Ziel war es, einen Überblick über die bestehenden Ressourcen zu schaffen, Belastungsfaktoren zu identifizieren und sowie Handlungsempfehlungen für den öffentlichen Gesundheitsdienst und die Flüchtlingshilfe zu entwickeln.

\section{Methodik}

Es wurden qualitative Befragungen mit 10 ehrenamtlichen Helferinnen und Helfern, sowie 11 koordinativ in der Flüchtlingshilfe tätigen Fachkräften durchgeführt. Die Befragungen fanden im Rahmen zweier Bachelorarbeiten von Ende April bis Ende Mai 2016 statt $[17,18]$.

Ehrenamtliche Interviewpartner/innen wurde mit Hilfe des Bezirksamt Hamburg-Bergedorf und in der Flüchtlingshilfe tätigen Vereinen über Rundschreiben, Emails, sowie soziale Medien wie Facebook, angeworben. Eine genaue Zahl der angesprochenen bzw. angeschriebenen lässt sich daher nicht angeben. Die Befragten waren in Flüchtlingsunterkünften in verschiedenen Stadtteilen Hamburgs tätig. Interviewt wurden Personen, die sich aufgrund der Ansprache freiwillig meldeten.

In der Gruppe der Expertinnen und Experten wurden Menschen befragt, die mit Ehrenamtlichen in der Flüchtlingshilfe zusammen arbeiten und deren Arbeit koordinieren. Im Einzelnen waren das Mitarbeitende der Bezirksämter (Koordination der sozialräumlichen Integration von Flüchtlingsunterkünften), Mitarbeitende des Sozialmanagements der Wohnunterkünfte für Flüchtlinge, darüber hinaus hauptamtliche Vertreter der Kirchengemeinden, die das ehrenamtliche Engagement der Gemeindemitglieder koordinieren, sowie die Koordinatorinnen und Koordinatoren von Ehrenamtsaufgaben in Organisationen der Flüchtlingshilfe. Erste Personen wurden auf Vorschlag von in der Ehrenamtskoordination tätigen Mitarbeitern des Bezirksamtes Hamburg-Bergedorf kontaktiert. Weitere Personenvorschläge kamen durch die ersten geführten Interviews zustande. Insgesamt wurden 13 potentielle Interviewpartner kontaktiert und es konnten elf Interviews durchgeführt werden.

Sowohl die befragten Ehrenamtlichen wie auch die befragten Expertinnen und Experten stellen ein Convenience-Sample dar, sind also weder systematisch, noch repräsentativ ausgewählt.

Die qualitativen Interviews mit den Ehrenamtlichen und den Expertinnen und Experten wurden anhand von Interviewleitfäden mit vergleichbaren Fragen geführt (siehe Anhang). Fragen wurden zu 
verschiedenen Themenblöcken in den Bereichen gesundheitliche Auswirkungen und Unterstützungsmöglichkeiten für Ehrenamtliche gestellt. Die Interviews wurden aufgenommen, transkribiert und mittels qualitativer Inhaltsanalyse nach Mayring [23] kategorisiert und ausgewertet.

Durch die qualitative Inhaltsanalyse sollten bedeutsame Einzelfaktoren und Zusammenhänge erkannt werden, um somit Gemeinsamkeiten in den Interviewtexten herauszuarbeiten. Für die Auswertung wurden die Interviews paraphrasiert und deduktive, an den Leitfaden und die Forschungsfrage angepasste Hauptkategorien als auch induktive, aus dem Material abgeleitete, Unterkategorien gebildet. Das hieraus resultierende Kategoriensystem ist in den $\triangleright$ Tab. 1 und $\triangleright \mathbf{2}$ wiedergegeben. Zu den jeweiligen Ober- und Unterkategorien wurden Paraphrasen kodiert und Ankerbeispiele aus den Interviews herausgesucht. Nach diesem Durchlauf wurden im nächsten Schritt alle Interviews mit dieser Struktur kodiert. Für die Auswertung wurde mit dem Programm MAXQDA gearbeitet. Im nachfolgenden Ergebnisteil werden die nach Auffassung der Autoren zentralen Ergebnisse der Interviews zusammengefasst. Dabei sind Zitate von Interviewpartnern zu Erläuterung und Illustration in Kursivdruck eingefügt. Um kenntlich zu machen, von welcher Befragtengruppe die jeweiligen Aussagen stammen, sind die Ergebnisse mit „EA“ für Aussagen von Ehrenamtlichen und/oder mit „EXP“ für die von Expertinnen und Experten gekennzeichnet. Auf die Häufigkeitsangaben hinsichtlich verschiedener Nennungen wurde bewusst verzichtet, da dies dem Charakter und der Aussagekraft einer qualitativen Befragung widerspricht. Eine qualitative Befragung kann und will nur darüber Auskunft geben, ob bestimmte Inhalte genannt wurden. Wie häufig bestimmte Inhalte relevant sind, müsste in darauf aufbauenden, weiterführenden Studien untersucht werden [23].

\section{Ergebnisse}

Die 10 befragten Ehrenamtlichen waren zwischen 54 und 69 Jahren alt. Von ihnen waren 7 weiblich und 3 männlich. Von den befragten Fachkräften waren vier weiblich und sieben männlich. Das Alter lag zwischen 26 und 65 Jahren.

\section{Motivation zu ehrenamtlicher Flüchtlingsarbeit}

Viele Ehrenamtliche sind aufgrund ihrer humanitären Grundeinstellung tätig (EA + EXP). Sie wollen etwas Gutes tun und Vorurteile abbauen. Einige von ihnen suchen auch eine Aufgabe in ihrem Leben, da sie schon älter und nicht mehr berufstätig sind (EA). Die aktuelle Lebenssituation bzw. deren Veränderung spielt dabei eine wichtige Rolle: Das Erwachsenwerden der Kinder oder der Einstieg in das Rentenalter, ist für die Aufnahme eines Ehrenamtes oft ausschlaggebend gewesen (EA).

\section{Positive Auswirkungen}

Alle befragten Ehrenamtlichen sehen ihr Ehrenamt als insgesamt positiv an und haben das Gefühl, dass sich ihr Wohlbefinden durch die Tätigkeit verbessert. „Also ich kann nur sagen, also für mich ist es echt, also es ist mir so wichtig, es ist ein unglaublich großes Glück. Eine Bereicherung, eine Freude und ich bin so froh, dass ich das gemacht habe“ (EA). Das Gebraucht-Werden und die erlebte Dankbarkeit und Wertschätzung wurden als stärkste positive Auswirkung auf
- Tab. 1 Kategoriensystem für die Auswertung der Interviews mit den Ehrenamtlichen.

- Gründe für das Engagement

- Direkte Anlässe

- Persönliche Ansprache

- Öffentlichkeitsarbeit des Vereins

- Motivationen und Beweggründe

- Aktuelle Lebenssituation

- Helfende Persönlichkeit

- Mitgestaltung der Gesellschaft

- Eigennützigkeit

- Pflichtbewusstsein

- Persönlicher Bezug

- Positive Auswirkungen und Ressourcen

- Individueller Mehrwert

- Sinnhaftigkeit

- Bestätigung von Anderen

- Zusammenarbeit mit Flüchtlingen

- (Kulturelle) Bereicherung

- Zusammenarbeit mit anderen Ehrenamtlichen

- Negative Aspekte und Belastungen

- Strukturelle Rahmenbedingungen

- Unterkunftsbedingte Schwierigkeiten (uneinheitliche Standards; Organisation von Seiten der Unterkunft; Regeln und Vorschriften)

- Zusammenarbeit mit Einrichtungsmitarbeitern (Kommunikationsschwierigkeiten; fehlende Wertschätzung; Hierarchiestrukturen; Arbeit der Einrichtungsmitarbeitenden)

- Zusammenarbeit mit Behörden und Institutionen (Koordination der Flüchtlingssituation; fehlende Wertschätzung; Überforderung der Mitarbeitenden)

- Zwischenmenschliche Konflikte

- Zusammenarbeit der Ehrenamtlichen (Kommunikationsschwierigkeiten; kulturelle Konflikte; Hierarchiestrukturen; fehlende Zuverlässigkeit)

- Zusammenarbeit mit Flüchtlingen (Sprachbarriere; kulturelle Schwierigkeiten)

- Gesundheitliche Auswirkungen

- Psychische Belastungen (zeitliche Belastung; ständige Präsenz der Problematik, hohe Verantwortung; Belastung der Flüchtlinge; persönliche Faktoren; Ausweglosigkeit der Situation; Gesamtsituation der Flüchtlingskrise)

- Umgang mit Belastungen

- Wissen über Unterstützung

- Individuelle Bewältigungsstrategien (Fort- und Weiterbildungen; Hilfe bei Anderen suchen; Ausgleich schaffen; Grenzen setzen)

- Verbesserungswünsche und Vorschläge der Befragten

- Organisation der Flüchtlingssituation

- Rahmenbedingungen der Aktivitäten

- Strukturierung des Ehrenamts

- Weiterbildungsangebote

die Ehrenamtlichen wahrgenommen. „Man bekommt so viel, von so vielen Menschen, [...] die einem dann auch was zurückgeben, wenn man etwas tut. [...]vom Gefühl her ist es unglaublich“ (EA). Das Ken- 
- Tab. 2 Kategoriensystem für die Auswertung der Interviews mit den Experten.

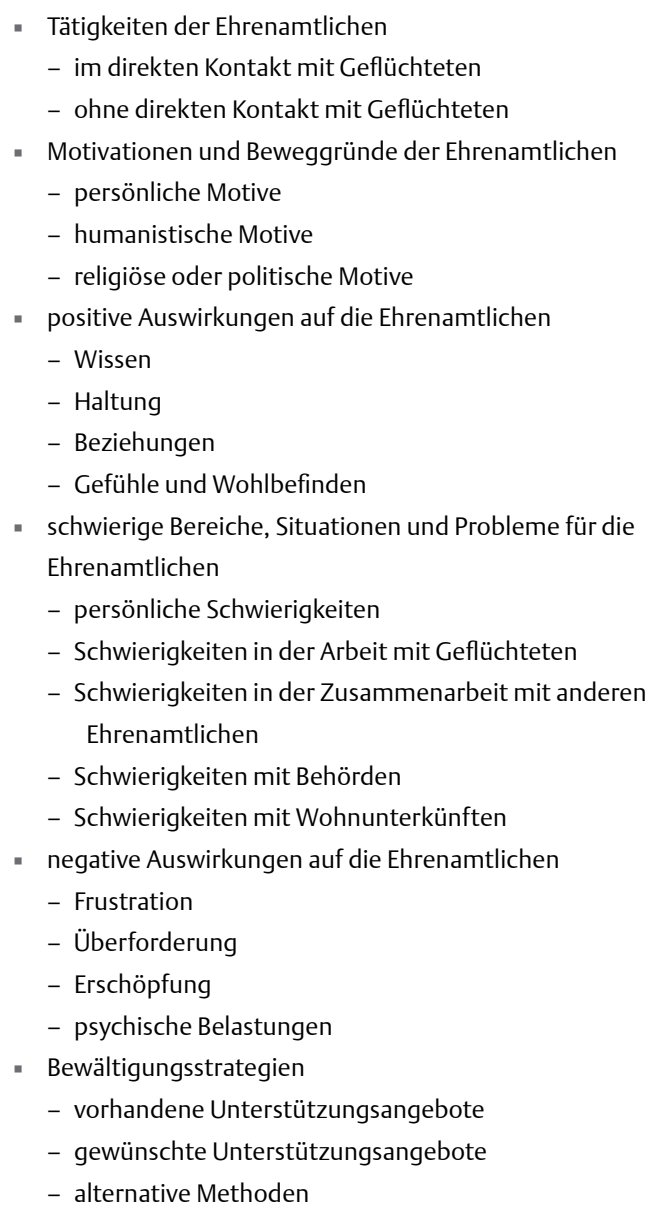

nenlernen neuer kultureller Aspekte genauso wie das Miteinander mit den Flüchtlingen und die Zusammenarbeit mit anderen Ehrenamtlichen wird als Bereicherung erlebt und neue Bekanntschaften und Freundschaften entstehen (EA).

Auch die befragten Expertinnen und Experten sehen viele positive Auswirkungen bei den Ehrenamtlichen. Genannt werden u.a.eine erhöhte Offenheit gegenüber Fremden, der Abbau von Vorurteilen und die Steigerung des Selbstbewusstseins (Haltung), engeres Gemeinschaftsgefühl im Stadtteils oder der Gemeinde, neue Freundschaften und die Abnahme von Einsamkeit (Beziehungen).

\section{Belastungen für die Ehrenamtlichen}

Neben den positiven Aspekten wurden aber auch Probleme und Belastungen beschrieben. Eine Befragte gab beispielsweise an: „Also ich denke Tag und Nacht daran und das ist mein erster Gedanke beim Aufwachen“ (EA). Eine andere Befragte berichtete: „Doch, es kommt ganz viel zurück. Ganz viel. Aber es ist auch super anstrengend. Und auch sehr belastend“ (EA). Manche Befragte erleben auch solche Belastungen, können sich aber gut abgrenzen: „a, das finde ich belastend, aber ich glaube jetzt nicht in der Form, dass es mich jetzt wirklich richtig tiefgehend belastet" (EA).

\section{Wo treten Belastungssituationen auf?}

Die Experten haben in den Interviews Probleme in verschiedenen Kategorien beschrieben ( $\triangleright$ Tab. 2).

Persönliche Schwierigkeiten treten durch Mangel an Fachwissen und Fertigkeiten für bestimmte Aufgaben auf und durch eine zu hohe Selbsterwartung. Dass dann auftretende Schwierigkeiten und Probleme von Ehrenamtlichen nicht artikuliert werden, wird als sehr problematisch wahrgenommen. Diese Punkte wurden ausschließlich in den Interviews mit den Expertinnen und Experten genannt.

Die befragten Expertinnen und Experten und die Ehrenamtlichen selbst nehmen Koordinations- und Kommunikationsprobleme mit Behörden, den Wohnunterkünften, unter den Ehrenamtlichen selbst sowie in der Betreuung der geflüchteten Menschen als belastend wahr. So führen bürokratische Hürden, lange Wartezeiten und der unklare Status von Geflüchteten zu Unverständnis und Frustration: „Es gibt Frustrationen bei den Ehrenamtlichen, es gibt Frustrationen bei den Bewohnerinnen und Bewohnern. Weil die im ersten Moment mit so viel Enthusiasmus und Engagement hier gestartet sind, [...]. Und sie sind fast ausnahmslos alle nicht weiter gekommen, weil sie nicht weiter kommen können. [...] Die wollen über das Anerkennungsverfahren, aber die kriegen nicht mal eine Einladung dazu. “ (EA).

Weitere Belastungen sind in > Tab. 1 zusammenfassend dargestellt. In der Zusammenarbeit mit den Geflüchteten berichten die Ehrenamtlichen auch von Unsicherheit, die durch das fehlende Wissen über die andere Kultur entsteht und dass sie Schwierigkeiten damit haben, die verschiedene Aspekte der Lebensweise und Gepflogenheiten anderer Kulturen zu akzeptieren: „Also sowas wie Pünktlichkeit und Zuverlässigkeit und ja, Verabredungen einhalten und überhaupt Initiative ergreifen oder was mitmachen. Das erlebe ich sehr häufig als sehr frustrierend, dass das so schwierig ist. Und dass die schwierige Aufgabe für mich dabei ist, das eben zu akzeptieren, dass andere Menschen anders leben" (EA).

\section{Gesundheitliche und psychische Belastungen}

Einige der Ehrenamtlichen gaben auf die Frage nach negativen gesundheitlichen Auswirkungen an, keine gesundheitlichen Belastungen wahrzunehmen. Andere hingegen bezeichneten ihr Ehrenamt als kräftezehrend, belastend und anstrengend.

Abgrenzungsprobleme, zeitliche wie emotionale, werden von fast allen Experten als das Hauptproblem in der direkten Arbeit der Ehrenamtlichen mit den Geflüchteten gesehen

Manche Ehrenamtliche können sich schlecht zeitliche Grenzen setzen und engagieren sich sehr viele Stunden, so dass kaum mehr Zeit für andere Dinge in ihrer Freizeit bleibt oder das private Umfeld vernachlässigt wird: „Und ansonsten ist es unheimlich übergriffig in mein Privatleben. Weil, also dass ich tatsächlich meine privaten Freundschaften ganz doll vernachlässigt habe“ (EA).

Schwierigkeiten mit der emotionale Abgrenzung entstehen durch die ständige Präsenz der Probleme der Geflüchteten (traumatisierende Erlebnisse, Hilflosigkeit mit der neuen Lebenssituation in Deutschland) einerseits und die Verantwortung, die die Ehrenamtlichen zu tragen haben andererseits. Einige Ehrenamtliche können mit diesen Schicksalen umgehen, für andere ist es in den Augen der Experten psychisch sehr belastend. „[...] wenn so ein Flüchtling plötzlich so viel Vertrauen gefasst hat, dass er was erzählt. 
Es gibt Leute, die können damit umgehen, wir haben relativ viele pädagogisch auf die eine oder andere Weise geschulte Leute unter den Ehrenamtlichen, aber nicht alle können damit umgehen." (EXP). Auch der aus der Abschiebung von Flüchtlingen resultierende Verlust von Freundschaft oder Bekanntschaft wird als Belastung genannt: „Oder manchmal ist es auch so, dass Familien auch einfach über Nacht dann weg sind, ohne dass man vorher von der Abschiebung wusste, und das macht natürlich auch vielen zu schaffen, weil dann auf einmal so ein Loch in die Gemeinschaft gerissen wird. [...] Und je enger der Kontakt, desto belastender ist das natürlich“ (EXP).

\section{Überforderung und Erschöpfung}

Von vielen Experten werden Ehrenamtliche beobachtet, die sich durch ihre Tätigkeit in der Flüchtlingshilfe überfordern. Die Helfer nehmen zu viele Aufgaben an und wollen mehr schaffen, als sie leisten können:. „Dass manch einer vielleicht dadurch, dass er die Not sieht, mehr versucht an Aufgaben zu übernehmen, als er selbst leisten kann“ (EXP). In der Folge dieser Überforderung kommt es auch zur Erschöpfung: „[...] aber es ist schon ein erheblicher Teil der Ehrenamtlichen, die sich bis zur absoluten Erschöpfung verausgaben. Und zwar weil sie ja mit Einzelschicksalen oder mit Einzelgeschichten Kontakt bekommen“ (EXP).

\section{Unterstützungsangebote}

Im Ganzen fühlen sich die befragten Ehrenamtlichen gut über Unterstützungsangebote informiert und wissen, an wen sie sich im Bedarfsfall wenden können. Sie fühlen sich durch die Anbindung an Flüchtlingsorganisationen gut aufgehoben; die regelmäßige Möglichkeit, Fragen klären zur können, und mit Information versorgt zu werden, wird als entlastend wahrgenommen.

Dennoch gaben in den Interviews mehrere Ehrenamtliche an, dass sie zwar von Unterstützungsmöglichkeiten wissen, aber noch keine Veranstaltungen besucht haben, da es ihnen an der Zeit fehlt, diese zu besuchen und das Angebot aufgrund der hohen Nachfrage derzeit noch nicht ausreicht.

Von Seiten der Expertinnen und Experten wird ein zusätzlicher Unterstützungsbedarf für die Helfer im Hinblick auf den Umgang mit psychischen Belastungen und die Abgrenzungsproblematik gesehen. Gleichzeitig merken sie aber auch an, dass bereits vorhandene Angebote von den Ehrenamtlichen oft nicht angenommen werden. Sie berichten, dass es ihnen noch nicht gelungen ist, mehr Ehrenamtliche zu Fortbildungen, Einzelgesprächen und Supervisionen zu ermutigen. Sie ermuten, dass die Ehrenamtlichen psychische Unterstützung aufgrund von Vorurteilen ablehnen und die Motivation, „anderen etwas Gutes zu tun“, sie davon abhält, sich mit ihrer eigenen Gesundheit zu befassen.

\section{Individuelle Bewältigungsstrategien}

Alle befragten Ehrenamtlichen haben bereits verschiedene individuelle Möglichkeiten gefunden, mit den Belastungen und Schwierigkeiten, mit denen sie sich konfrontiert sehen, umzugehen $>$ Tab. 1.

Einigen der Interviewten gelingt es offenbar, die oben angesprochene Abgrenzungsproblematik für sich zumindest teilweise lösen zu können. Sie gaben an, sich bewusst Grenzen zu setzen und zu gewissen Zeiten von der Ehrenamtstätigkeit Abstand zu nehmen. Dieser Aspekt wird jedoch gleichzeitig als sehr herausfordernd empfunden. Manche der Ehrenamtlichen berichteten auch, dass sie es erst mit dem Ehrenamt gelernt haben, Nein zu sagen und Aufgaben abzulehnen; zudem gaben einige Befragte an, zwar in der Theorie zu wissen, dass sie sich stärker abgrenzen müssen, dies jedoch in der Praxis nur schwer umsetzen können. „Ich denke, was ich, was jede Freiwillige lernen muss, ist abgrenzen. Das finde ich, das ist das aller schwerste. Vor allen Dingen, wenn da diese persönlichen Bindungen entstanden sind" (EA).

\section{Strukturelle Hilfen, Angebote}

Neben Änderungswünschen in der Zusammenarbeit mit Behörden, zu den Abläufen in Wohnunterkünften und zu politischen Verbesserungen insgesamt wurden auch konkrete Anregungen zu Weiterbildungsangeboten durch die Ehrenamtlichen gemacht. Das betraf Weiterbildungsangebote zu Asylrecht und Behördenstrukturen, aber auch Wissen im Umgang mit Traumatisierung von Flüchtlingen und Verbesserung der interkulturellen Kompetenz.

Angebote zum Thema Selbstfürsorge für Ehrenamtliche wurden ebenfalls gewünscht. „Und vielleicht auch sogar, weiß ich nicht, so eine Art Seminarangebot,Umgang mit persönlichen Belastungen durch die Geschichten von Flüchtlingen ' und so. Denn man nimmt schon auch Nachrichten, also ich lese die Zeitung anders, ich nehme die Nachrichten anders wahr, wenn man einen Jungen aus Aleppo kennt oder aus Kundus oder aus Eritrea, wenn man weiß, was da los ist“ (EA).

Eine andere Anregung war die Einrichtung einer Beratungsstelle, an die sich Ehrenamtliche wenden können, um über ihre Belastungen und Sorgen zu sprechen. Diese Stelle sollte laut den Befragten unabhängig von den Trägern der Flüchtlingsarbeit sein, damit auch Kritik unbefangen geäußert werden kann.

\section{Unterstützungsangebote zum Zeitraum der Interviews}

Zum Zeitpunkt der Interviews bestanden in Hamburg Angebote von Fortbildungen und Workshops, die vom Thema Asylrecht über interkulturelle Kompetenzen bis hin zum Thema Erkennung von Traumata reichen. Dennoch scheint das Angebot, gemessen an der Vielzahl von Ehrenamtlichen, die allein in Hamburg tätig sind, nicht sehr groß zu sein. Hinzu kommt, dass die Fortbildungen in der Regel nur einmal stattfinden und der Veranstaltungsort teilweise am Rande der Stadt liegt. Zudem sind einige der Fortbildungen kostenpflichtig, worin eine weitere Teilnahmebarriere liegt. Auch wenn diese Kosten übernommen werden könnten, fehlt oft die Information darüber, wer welche Maßnahmen bezahlt oder bezuschusst. Ein systematisches und kontinuierliches Angebot, mit dem die Ehrenamtlichen im Umgang mit belastenden Situationen unterstützt werden, existiert nicht, auch wenn die Mitarbeiter von Unterkunftsbetreibern für Gespräche zur Verfügung stehen.

\section{Diskussion}

Die qualitativen Interviews von ehrenamtlich in der Flüchtlingshilfe tätigen Menschen sowie von Fachleuten mit koordinierenden Tätigkeiten in der Flüchtlingshilfe haben gezeigt, dass das Ehrenamt von vielen Freiwilligen als eine starke Ressource erlebt und genutzt werden kann. Viele Menschen erfahren durch die ehrenamtliche Tätigkeit eine Steigerung ihres Wohlbefindens und ihres Selbstwerts. Gleichzeitig erleben jedoch manche, aber nicht alle 
Ehrenamtlichen auch zeitliche und emotionale Belastungen, die zu gesundheitlichen und psychischen Belastungen führen. Als wichtigste Ressource für den Umgang mit diesen Belastungen wurde immer wieder die Fähigkeit zur Abgrenzung genannt. Während manche Ehrenamtliche diese Fähigkeit bereits mitbringen oder in ihrer Tätigkeit entwickeln, haben andere hier noch einen Entwicklungsbedarf.

Bei der Interpretation der Ergebnisse dieser Befragungen muss berücksichtigt werden, dass es sich um qualitative Interviews mit explorativem Charakter handelt. Aus den hier berichteten Befragungsergebnissen kann somit immer nur geschlossen werden, dass die genannten Gesichtspunkte für einige Befragte relevant sind. Rückschlüsse darüber, ob diese Gesichtspunkte nur für wenige oder für viele Betroffene relevant sind, können mit einem solchen Forschungsansatz nicht gezogen werden. Hinzu kommt, dass sowohl die interviewten Freiwilligen als auch die interviewten Expertinnen und Experten ein kleines Convenience-Sample darstellen, das weder auf einer Zufallsauswahl noch auf einer systematischen Auswahl beruht und daher keinen Anspruch auf Repräsentativität erheben kann. Da die interviewten Personen aber nach eigenen Angaben zur Zielgruppe der ehrenamtlich in der Flüchtlingshilfe tätigen Personen gehörten, bzw. zur Fachleuten mit koordinierenden Tätigkeiten in der Flüchtlingshilfe, kann davon ausgegangen werden, dass die berichteten Ergebnisse für die Zielgruppe relevant sind.

Vor diesem Hintergrund können aus den Ergebnissen die folgenden, vorläufigen Schlussfolgerungen und Handlungsoptionen abgeleitet werden.

\section{Handlungsmöglichkeiten und Konsequenzen}

Bei einem ehrenamtlichen Engagement kommt es auf strukturelle Bedingungen an, die dafür sorgen, dass Ehrenamtliche in einem angemessenen zeitlichen Rahmen arbeiten, sich nicht überfordern, auf ihre Aufgaben vorbereitet werden und durch regelmäßige Supervisionen unterstützt werden [19].

Um geflüchtete Menschen zu unterstützen, ist es für die Ehrenamtlichen wichtig, sich über die eigenen Ressourcen aber auch über die Belastungen, die mit der ehrenamtlichen Tätigkeit einhergeht, bewusst zu sein.

Hier wäre zu prüfen, ob der Öffentliche Gesundheitsdienst ÖGD entsprechende Angebote entwickeln und umsetzen kann. In den meisten Sozialpsychiatrischen Diensten der Gesundheitsämter arbeitet ein multiprofessionelles Team mit Zusatzausbildungen in systemischer Beratung, Therapie und Supervision. Des Weiteren haben Mitarbeiter/innen in der (kommunalen) Gesundheitsförderung vielfältige Erfahrungen zu Unterstützungsangeboten, Programmen und Trainings, die im Wesentlichen darauf abzielen, Zielgruppen zu stärken.

Angebote zur Supervision, runde Tische für die Ehrenamtlichen mit den Themen Abgrenzungsprobleme oder Umgang mit Kommunikationsschwierigkeiten aber auch die Steigerung der Selbstwirksamkeit für einen konstruktiven Umgang mit Stress, sowie Selbstfürsorge und Achtsamkeit sind Maßnahmen, die zur Bewältigung der Belastungen und psychischen Problemen, denkbar sind.

Hierbei können Teile/einzelne Abteilungen des ÖGD, Maßnahmen in Zusammenarbeit mit Flüchtlingsorganisation vor Ort anregen und vermitteln und wenn möglich, selber durchzuführen. In
Hamburg Bergedorf sind aufgrund der hier dargestellten Ergebnisse, erste Maßnahmen umgesetzt worden. Auf einem Workshop zur Ressourcenförderung sind zusammen mit Ehrenamtlichen, Ressourcen und Belastungen in der Arbeit mit Geflüchteten ermittelt worden. Als Ergebnis konnten umgehend konkrete Arbeitsaufgaben an die örtliche Flüchtlingsorganisation adressiert und Fortbildungsmaßnahmen zur Selbstfürsorge verabredet werden. Darüber hinaus nahmen Ehrenamtliche zusammen mit Mitarbeitern aus dem Gesundheitsamt an einer sogenannten Community Resiliency Model (CRM)- Lotsenschulung teil [24].

Weiterhin steht dem ÖGD das Instrument der Gesundheitskonferenzen zur Verfügung, auf denen Projekte und Maßnahmen im Bereich der Gesundheitsförderung von Geflüchteten und Ehrenamtlichen vorgestellt werden können [25].

Allerdings ist auch schon ein breites Angebot an Unterstützung für Ehrenamtliche vorhanden. Diese Unterstützungsangebote werden jedoch aus den verschiedensten Gründen nicht in der gewünschten Form von der Zielgruppe angenommen. Die Experten bemängeln in diesem Zusammenhang vor allem die Kommunikationsschwierigkeiten und undurchsichtige Strukturen, sodass davon auszugehen ist, dass viele Informationen über die Angebote oftmals nicht bei den ehrenamtlich Tätigen ankommen.

Um die Teilnahmequote an Supervisionen und psychischen Unterstützungsangeboten zu erhöhen, müssten die Gründe für das Fernbleiben von solchen Angeboten genauer untersucht werden. Damit Vorurteile und Ängste gegenüber psychischer Unterstützung zu verringert werden können, ist eine umfangreiche Aufklärung über die Angebote und deren Nutzen sinnvoll. Auch hierin könnte eine wichtige Aufgabe des ÖGD liegen.

In Extremfällen, z. B. für besonders belastete Gruppen von Ehrenamtlichen, die sich zeitlich sehr stark einbinden oder psychisch durch ihre Tätigkeiten ausgesprochen belastet werden, könnte eine verpflichtende Teilnahme an Fortbildungen und Supervisionen diskutiert werden. Dabei stellt sich natürlich die Frage, wie und durch wen solche besonderen Bedarfe erkannt werden können und wie dann sinnvolle Unterstützungsmaßnahmen rechtzeitig eingeleitet werden können.

Last, not least wäre zu überlegen, ob der ÖGD in der Zusammenarbeit der verschiedenen behördlichen Strukturen unterstützend tätig werden kann.

Insgesamt zeigt sich, dass das Thema des Ehrenamtes in der Flüchtlingshilfe nicht ausreichend wissenschaftlich untersucht ist. Es ist also weitere Forschung, insbesondere hinsichtlich der gesundheitlichen Auswirkungen, dringend notwendig, um die Ehrenamtlichen vor Belastungen zu schützen, aber auch um die Ressourcen, die im Ehrenamt entwickelt werden, besser nutzen zu können. Unter anderem wäre hierbei zu untersuchen, welche Faktoren die psychische Widerstandsfähigkeit der Helfer stärken können (Resilienzfaktoren), ob eine verbesserte Selbstwirksamkeit bei der Bewältigung von Belastungen hilfreich ist, und wie letztlich solche hilfreichen Ressourcen systematisch aufgebaut und verstärkt werden können. Es ist geplant, aufbauend auf den hier berichteten qualitativen Ergebnissen einen Fragebogen zu entwickeln, mit dem gesundheitliche Ressourcen und Belastungsfaktoren in der ehrenamtlichen Flüchtlingshilfe auch quantitativ abgeschätzt werden können. 
Letztlich wurde in unserer Befragung auch das enorme Potenzial deutlich, das im Wissen und der Erfahrung der Ehrenamtlichen steckt. Die Freiwilligen sollten deutlich mehr in die verschiedenen Prozesse der Flüchtlingshilfe eingebunden werden, da sie sowohl im Kontakt mit den Flüchtlingen selbst als auch im Austausch mit den Unterkunftsleitungen stehen und somit Probleme aus mehreren Perspektiven wahrnehmen.

\section{Interessenkonflikt}

Die Autoren geben an, dass kein Interessenkonflikt besteht.

Literatur

[1] UNHCR. UNHCR - Figures at a Glance [Internet]. [zitiert 19. Oktober 2016]. Verfügbar unter: http://www.unhcr.org/figures-at-a-glance.html

[2] Bundesamt für Migration und Flüchtlinge. Aktuelle Zahlen zu Asyl. Ausgabe September 2016 [Internet]. 2016 Sep [zitiert 19. Oktober 2016]. Verfügbar unter: http://www.bamf.de/SharedDocs/Anlagen/ DE/Downloads/Infothek/Statistik/Asyl/aktuelle-zahlen-zu-asyl-september-2016.pdf?_blob = publicationFile

[3] Landeszentrale für politische Bildung Baden-Württemberg. . Flüchtlingsproblematik. Flüchtlinge in Deutschland [Internet]. [zitiert 19. Oktober 2016]. Verfügbar unter: http://www.lpb-bw.de/ fluechtlingsproblematik.html\#c24499

[4] Statista. EU - Illegale Grenzübertritte durch Flüchtlinge nach Fluchtrouten 2016 | Statistik [Internet]. [zitiert 28. Oktober 2016]. Verfügbar unter: https://de.statista.com/statistik/daten/studie/461364/umfrage/illegale-grenzuebertritte-in-die-eu-durchfluechtlinge-nach-fluchtrouten/

[5] Bendel P.Herausgeber Was Flüchtlinge brauchen - Ein Win-Win-Projekt. Ergebnisse aus einer Befragung in Erlangen. Erlangen: FAU University Press; 2016

[6] Sphere Project. The Sphere Project. Bonn: Köllen; 2011

[7] Gardemann J, Wilp T. Gültigkeit international verbindlicher, normativer und technischer Standards der Flüchtlingshilfe auch in den deutschen Erstaufnahmeeinrichtungen. Bundesgesundheitsblatt - Gesundheitsforschung - Gesundheitsschutz 2016; 59: 556-560

[8] Razum O, Saß A-C, Bozorgmehr K. Gesundheitliche Versorgung von Geflüchteten: Herausforderungen und Lösungsansätze. Bundesgesundheitsblatt - Gesundheitsforschung - Gesundheitsschutz 2016; 59: 543-544

[9] Wiesmüller G, Dötsch J, Weiß M, Wiater A, Fätkenheuer G, Nitschke H. u. a. Kölner Statement zur medizinischen Versorgung von Flüchtlingen. Gesundheitswesen 2016; 78: 237-238

[10] Tinnemann P, Gundlach F, Nitschke H, Bunte A, Teichert U. Medizinische Versorgung von Flüchtlingen durch den Öffentlichen Gesundheitsdienst: Allzeit bereit - nur wie lange noch? Gesundheitswesen 2016; 78: 195-199

[11] Karakayali S, Kleist JO. Strukturen und Motive der ehrenamtlichen Flüchtlingsarbeit (EFA) in Deutschland - 1. Forschungsbericht; Ergebnisse einer explorativen Umfrage vom November/Dezember 2014. [Internet]. Berlin: Berliner Institut für empirische Integrationsund Migrationsforschung. Humboldt-Universität zu Berlin; 2015 [zitiert 18. Mai 2016]. Verfügbar unter: http://www.bim.hu-berlin.de/ media/2015-05-16_EFA-Forschungsbericht_Endfassung.pdf
[12] Borkowski A.. „Ohne Ehrenamtliche wären wir aufgeschmissen“ - Angebot und Netzwerkstrukturen der Erlanger Flüchtlingsarbeit. In: Bendel P. Herausgeber Was Flüchtlinge brauchen - ein Win-Win-Projekt Ergebnisse aus einer Befragung in Erlangen. Erlangen: FAU University Press; 2016

[13] Han-Broich M. Engagement in der Flüchtlingshilfe - eine Erfolg versprechende Integrationshilfe. Aus Polit Zeitgesch 2015; 65: 43-49

[14] Bundesministerium für Familie, Senioren, Frauen und Jugend. Monitor Engagement. Ausgabe Nr. 2. Freiwilliges Engagement in Deutschland 1999 -2004 - 2009. Ergebnisse der repräsentatien Trenderhebung zu Ehrenamt, Freiwilligenarbeit und bürgerschaftlichem Engagement. [Internet]. Berlin; 2010 Apr [zitiert 23. Mai 2017]. Verfügbar unter: https://www.bmfsfj.de/blob/94370/41b89528c91dc57f95ec842398b a581c/monitor-engagement-nr-2-data.pdf

[15] Bundesministerium für Familie, Senioren, Frauen und Jugend. Hauptbericht des Freiwilligensurveys 2009. Zivilgesellschaft, soziales Kapital und freiwilliges Engagement in Deutschland 1999 - 20004 - 2009 [Internet]. Berlin; 2010 Okt [zitiert 17. Mai 2017]. Verfügbar unter: https://www.bmfsfj.de/blob/jump/93170/3--freiwilligensurveyhauptbericht-data.pdf

[16] Winkler J. Über das Ehrenamt. 1. AuflBremen: Europ. Hochsch.-verl; 2011: 133 S. (Wismarer Schriften zu Management und Recht)

[17] Borkel V. Gesundheitliche Belastungen und Unterstützungsbedarfe von Ehrenamtlichen in der Flüchtlingshilfe. Experteninterviews mit Personen mit koordinativen Aufgaben. [Bachelorarbeit]. Hamburg: Hochschule für Angewandte Wissenschaften Hamburg, Department Gesundheitswissenschaften; 2016

[18] Freyer B. Unterstützungsbedarfe von Ehrenamtlichen in der Flüchtlingshilfe und gesundheitliche Auswirkungen des Ehrenamts. Qualitative Interviews mit ehrenamtlich Tätigen. [Bachelorarbeit]. Hamburg: Hochschule für Angewandte Wissenschaften Hamburg, Department Gesundheitswissenschaften; 2016

[19] Warner LM. „Wer andern hilft, der hilft auch sich selbst“ - Wie Helfen Zufriedenheit und Gesundheit fördern kann. Informationsdienst Altersfragen 2009; 36: 2-26

[20] Bundesministerium für Familie, Senioren, Frauen und Jugend. Monitor Engagement. Wie und wofür engagieren sich ältere Menschen. Ausgabe Nr. 4 [Internet]. Berlin; 2011 März [zitiert 19. Oktober 2016]. Verfügbar unter: https://www.bmfsfj.de/blob/94372/390e22aa8a484 c4be0ae989d3de66ee5/monitor-engagement-nr-4-data.pdf

[21] Spitzer M. Editorial: Geben ist seliger denn Nehmen $(p<0.05)$ - Ehrenamt und Gesundheit. Nervenheilkunde 2006; 25: 994-996

[22] Brown SL, Nesse RM, Vinokur AD, Smith DM. Providing social support may be more beneficial than receiving it: results from a prospective study of mortality. Psychol Sci. Juli 2003; 14: 320-327

[23] Mayring P. Qualitiative Inhaltsanalyse: Grundlagen und Techniken. 12. AuflageWeinheim, Basel: Beltz; 2015

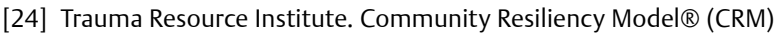
[Internet]. [zitiert 23. Mai 2017]. Verfügbar unter: http://traumaresourceinstitute.com/community-resiliency-model-crm/

[25] Bezirksamt Hamburg-Nord. Gesundheitskonferenz 2016 [Internet]. [zitiert 17. Mai 2017]. Verfügbar unter: ( http://www.hamburg.de/con tentblob/8340144/90a797f7ede32b3ff9e0355ee7673af8/data/ gesundheitskonferenz-2016-09-28.pdf 


\section{Anhang}

> Anhang 1 Gegenüberstellung der Interviewleitfäden.

\begin{tabular}{|c|c|}
\hline Interviews mit Ehrenamtlichen & Interviews mit Experten \\
\hline \multicolumn{2}{|c|}{$\begin{array}{l}\text { Vorstellung des Interviewers (Projektvorstellung, Studentin, Thema des Interviews, Dank für das Interview, geplante Dauer, Erlaubnis Tonbandaufnah- } \\
\text { me, auf Anonymität hinweisen) }\end{array}$} \\
\hline \multicolumn{2}{|l|}{ Vorstellung des Befragten (Geschlecht, Alter, Herkunft, Beruf, Familienstand) } \\
\hline & $\begin{array}{l}\text { Bitte beschreiben Sie ihre eigene Tätigkeit in der Flüchtlingshilfe (Tätigkeit, } \\
\text { Umfang/Stellenwert, Ort, Institution/Anbindung/Verortung, mit welchen } \\
\text { Personen/Zielgruppen, seit wann). }\end{array}$ \\
\hline $\begin{array}{l}\text { Bitte beschreiben Sie das Ehrenamt, das sie zurzeit ausführen } \\
\text { (Tätigkeit, Umfang, Ort, mit welchen Personen/Zielgruppen, seit } \\
\text { wann, Aufwandsentschädigung). }\end{array}$ & $\begin{array}{l}\text { Bitte beschreiben Sie die Tätigkeiten der ehrenamtlichen Flüchtlingshelfer, mit } \\
\text { denen Sie zusammen arbeiten (ggf. wichtigste Aspekte zusammenfassen: } \\
\text { Tätigkeit, Umfang, Ort, mit welchen Personen/Zielgruppen, seit wann, } \\
\text { Aufwandsentschädigung). }\end{array}$ \\
\hline $\begin{array}{l}\text { Wie sind Sie dazu gekommen, sich ehrenamtlich in der Flüchtlings- } \\
\text { hilfe zu engagieren? } \rightarrow \text { Motivation }\end{array}$ & $\begin{array}{l}\text { Welche Motivationen und Beweggründe können Sie bei den ehrenamtlichen } \\
\text { Flüchtlingshelfern für ihr Engagement erkennen? } \rightarrow \text { Motivation }\end{array}$ \\
\hline $\begin{array}{l}\text { Was gefällt Ihnen an Ihrem Ehrenamt? (Gibt es positive Auswirkun- } \\
\text { gen auf Ihr Wohlbefinden? Welche?) }\end{array}$ & $\begin{array}{l}\text { Welche positiven Auswirkungen hat das ehrenamtliche Engagement auf die } \\
\text { ehrenamtlichen Helfer und Helferinnen? (Gesundheit, Zufriedenheit, } \\
\text { Selbstbewusstsein, Wissen, usw.). }\end{array}$ \\
\hline \multicolumn{2}{|l|}{$\begin{array}{l}\text { Falls noch nicht genannt: Gibt es Bereiche, die Ihnen Kraft und } \\
\text { Energie geben und so zu mehr Gesundheit / größerer Zufriedenheit } \\
\text { beitragen? } \rightarrow \text { Gesundheitsaspekt }\end{array}$} \\
\hline $\begin{array}{l}\text { Was gefällt Ihnen an Ihrem Ehrenamt weniger gut? Gibt es Dinge, } \\
\text { die Sie stören? }\end{array}$ & $\begin{array}{l}\text { Was glauben Sie, in welchen Situationen und Bereichen die ehrenamtlichen } \\
\text { Helfer und Helferinnen am meisten Schwierigkeiten haben? (Wissen, Haltung, } \\
\text { Handeln: Überforderung, Kultur, Sprache...) }\end{array}$ \\
\hline $\begin{array}{l}\text { Gibt es Bereiche/Situationen, die Sie in Ihrer ehrenamtlichen } \\
\text { Tätigkeit als besonders belastend empfinden? Wenn ja, welche? }\end{array}$ & $\begin{array}{l}\text { Welche Schwierigkeiten treten für Sie und/oder für die Ehrenamtlichen in der } \\
\text { Zusammenarbeit auf? (Kommunikation, Zuverlässigkeit, Wissen, Haltung, } \\
\text { Handeln usw.). }\end{array}$ \\
\hline $\begin{array}{l}\text { Hat das Ehrenamt negative Auswirkungen auf Ihre Gesundheit? } \\
\text { Wenn ja, welche? (Somatisch / psychisch) Beispiele: } \\
\text { - Neigen Sie dazu sich zu überfordern? } \\
\text { - Setzen Sie sich genug Grenzen? }\end{array}$ & $\begin{array}{l}\text { Welche Auswirkungen hat die ehrenamtliche Tätigkeit auf die Gesundheit der } \\
\text { Helferinnen und Helfer? (Somatisch / psychisch) Beispiele: } \\
\text { - Neigen sie dazu sich zu überfordern? } \\
\text { - Setzen sie genug Grenzen? }\end{array}$ \\
\hline \multirow[t]{2}{*}{$\begin{array}{l}\text { Wenn Sie an die Sie belastenden Situationen denken, was könnte in } \\
\text { diesem Zusammenhang verbessert werden? Beispiele: mehr Entlastung, } \\
\text { mehr Fortbildung, mehr Anerkennung, Aufwandsentschädigung? } \\
\text { - Welche Unterstützungsmöglichkeiten kennen Sie? (Wissen) } \\
\text { - Was könnten Sie noch tun, um Ihr Wohlbefinden zu steigern? } \\
\quad \text { (Haltung) } \\
\text { - Tun Sie bereits etwas, um die Situation für sich selbst zu } \\
\text { verbessern? (Handeln) }\end{array}$} & $\begin{array}{l}\text { Was könnte in diesem Zusammenhang verbessert werden? Beispiele: mehr } \\
\text { Entlastung, mehr Fortbildung, mehr Anerkennung, Aufwandsentschädigung? }\end{array}$ \\
\hline & $\begin{array}{l}\text { Was könnten die Ehrenamtlichen selbst für sich tun? (sich Pausen gönnen, } \\
\text { Gesundheitskurse ...) }\end{array}$ \\
\hline \multirow[t]{2}{*}{$\begin{array}{l}\text { Welche Art von Unterstützung wünschen Sie sich für Ihre Tätigkeit } \\
\text { im Ehrenamt? In welchen Bereichen? Von wem? } \\
\text { - Gibt es Dinge, bei denen Sie das Gefühl haben, Sie müssten mehr } \\
\text { darüber wissen? (Wissen) } \\
\text { - Gibt es Situationen, in denen Sie das Gefühl haben, damit nicht } \\
\text { umgehen zu können? (Haltung) } \\
\text { - Gibt es Situationen, in denen Sie im Nachhinein denken „das hätte } \\
\text { ich anders machen sollen“? (Handeln) }\end{array}$} & $\begin{array}{l}\text { Welche Unterstützung gibt es für die ehrenamtlichen Helfer und Helferinnen in } \\
\text { dieser Situation? In welchen Bereichen? Was und von wem? } \\
\text { - Wissen (Fortbildungen, Kurse) } \\
\text { - Haltung (Änderung eigene Haltung, Haltung anderer) } \\
\text { - Handeln (finanzielle Unterstützung, Hilfsmittel, Zeit) } \\
\text { - Gesundheit (Kurse, Coaching, Hygiene...) } \\
\text { - Sicherheit (Hilfsmittel, Sicherheitseinweisungen...) }\end{array}$ \\
\hline & $\begin{array}{l}\text { Und was glauben Sie wäre zusätzlich sinnvoll? } \\
\text { - Wissen (Fortbildungen, Kurse) } \\
\text { - Haltung (Änderung eigene Haltung, Haltung anderer) } \\
\text { - Handeln (finanzielle Unterstützung, Hilfsmittel, Zeit) } \\
\text { - Gesundheit (Kurse, Coaching, Hygiene...) } \\
\text { - Sicherheit (Hilfsmittel, Sicherheitseinweisungen...) }\end{array}$ \\
\hline $\begin{array}{l}\text { Wenn Sie in Ihrer ehrenamtlichen Arbeit etwas verändern könnten, } \\
\text { was wäre das? (sehr offene Frage) }\end{array}$ & $\begin{array}{l}\text { Wenn Sie jetzt konkret etwas an Ihrer eigenen Arbeit in der Flüchtlingshilfe ändern } \\
\text { könnten, was würden Sie tun? (sehr offene Frage) }\end{array}$ \\
\hline \multicolumn{2}{|c|}{ Nachfragen/Unklarheiten vom Befragten \& weitere, noch nicht angesprochene Themen } \\
\hline Dank & \\
\hline
\end{tabular}

